\title{
Concentración foliar de macro- y micro-nutrimentos en cuatro leguminosas maderables del trópico estacionalmente seco de Costa Rica
}

\author{
Alfredo Alvarado ${ }^{1}$, Argenis Mora ${ }^{2}$, Eduardo Chacón-Madrigal ${ }^{3,4}$, José E. Villalobos \\ \& Carlos L. Sandí ${ }^{2}$ \\ 1. Centro de Investigaciones Agronómicas, Universidad de Costa Rica, San José, Costa Rica. \\ Correspondencia; alfredo.alvarado@ucr.ac.cr \\ 2. Universidad EARTH, Las Mercedes de Guácimo, Limón, Costa Rica. \\ 3. Escuela de Biología, Universidad de Costa Rica; 11501-2060 San José, Costa Rica. \\ 4. Faculty Centre of Biodiversity, University of Vienna, Rennweg 14, 1030 Viena, Austria.
}

Recibido 02-X-2017. Corregido 14-V-2018. Aceptado 13-VI-2018.

\begin{abstract}
Foliar concentration of macro- and micronutrients in four woody legumes from a tropical dry-seasonal forest in Costa Rica. The information about nutritional aspects of trees in the tropical dry forest is scarce. This investigation aims to establish a normal range of foliar nutrient concentration to four forestry species as related to their growth rates and biological nitrogen fixation capacity in the seasonally dry ecosystem at Guanacaste, Costa Rica. Foliar samples for four species: Dalbergia retusa (cocobolo), Enterolobium cyclocarpum (guanacaste), Hymenaea courbaril (guapinol or jatobá) and Schizolobium parahyba (gallinazo or pachaco), all belonging to the Fabaceae family, were analyzed for $\mathrm{N}, \mathrm{P}, \mathrm{Ca}, \mathrm{Mg}, \mathrm{K}, \mathrm{S}, \mathrm{Fe}, \mathrm{Mn}, \mathrm{Cu}, \mathrm{Zn}, \mathrm{B}$ and Na. Nutrient concentration varied with species according to their ability to fix nitrogen and their rate of growth. The overall concentration of macronutrients was higher in nitrogen-fixing species compared with non-fixing species, and within each one of those groups, the species with higher growth rate presented more macronutrients than species with low growth rate. According to the concentration of foliar nutrients accumulated in the foliage, we recommend that $H$. courbaril and $D$. retusa could be grown in the region soils with medium fertility and $E$. cyclocarpum and S. parahyba in the more fertile soils. Values ranges of foliar concentrations considered as satisfactory presented in this work are new for $D$. retusa and $H$. courbaril and improve the scarce information available for E. cyclocarpum and S. parahyba. Rev. Biol. Trop. 66(3): 969-983. Epub 2018 September 01.
\end{abstract}

Key words: foliar analysis; seasonal dry tropics; Dalbergia retusa; Enterolobium cyclocarpum; Hymenaea courbaril y Schizolobium parahyba.

Después de las gramíneas, las leguminosas son consideradas como la segunda familia de plantas en importancia económica. Esto debido al número de especies que proveen alimento para la población humana, forraje para el ganado, leña, diversos extractos o compuestos usados en la industria y a que ecológicamente incluyen una gran diversidad de especies y formas de vida (Lewis, 2005). Las leguminosas ocupan varios hábitats pero son particularmente dominantes en el bosque tropical estacionalmente seco (Gillespie, Grijalva, \& Farris, 2000; Jacobs, 2004). Algunas de ellas tienen la capacidad de fijar $\mathrm{N}$ a través de una interacción con bacterias del género Rhizobium, lo que les permite influir en la conformación de las comunidades vegetales y contribuir en la restauración de suelos degradados (Gei, 2014; Menge \& Chazdon, 2016).

Varias especies de leguminosas maderables del trópico americano se utilizan en plantaciones forestales debido a la alta densidad y coloración de su madera, resistencia a plagas y altas tasas de crecimiento (Piotto, Víquez, Montagnini, \& Kanninen, 2004). Sin embargo, existe poca información sobre los requerimientos nutricionales de las especies más utilizadas y si esto es afectado por su capacidad de fijar 
nitrógeno, su tasa de crecimiento o su tolerancia a la sombra. En el presente estudio se analizan los requerimientos nutricionales de las especies cocobolo (Dalbergia retusa, subfamilia Faboideae, fijadora de $\mathrm{N}$ y crecimiento lento), guanacaste (Enterolobium cyclocarpum, subfamilia Mimosoideae, fijadora de $\mathrm{N}$ y crecimiento rápido), guapinol (Hymenaea courbaril, subfamilia Caesalpiniodeae, no fijadora y de crecimiento lento) y gallinazo (Schizolobium parahyba, subfamilia Caesalpiniodeae, no fijadora y de crecimiento rápido). Estas especies son de uso común en plantaciones para producción de madera y pueden clasificarse en pares de acuerdo a la capacidad de fijar nitrógeno y a su tasa de crecimiento (Cuadro 1).

Los nutrimentos cumplen un papel esencial en la fisiología vegetal y cuando uno de ellos no se encuentra en las cantidades satisfactorias, su deficiencia en los tejidos promueve cambios en el metabolismo de la planta (Marschner, 1995). En su mayoría los elementos esenciales requeridos por las plantas se absorben vía raíces asociadas a micorrizas, unos en cantidades grandes a moderadas, llamados macronutrimentos (incluyendo los mesonutrimentos) como N, P, K y Ca, Mg y S. Un segundo grupo que se absorben en cantidades pequeñas y se conocen como micronutrimentos o elementos traza, entre ellos $\mathrm{Fe}, \mathrm{Si}, \mathrm{Cu}, \mathrm{Cl}, \mathrm{Mn}, \mathrm{B}, \mathrm{Zn}$ y Mo (Marschner, 1995).

Pocos valores de concentración de nutrimentos han sido reportados para hojas y madera de E. cyclocarpum y de S. parahyba (Cuadro 2). Para la especie $H$. courbaril, creciendo bajo condiciones de invernadero, se considera que las plántulas tienen un bajo requerimiento de $\mathrm{P}$ pues no responden a la adición del elemento (Resende, Neto, Muniz, Curi, \& Faquin, 1999; Resende, Neto, \& Curi, 2005) ni a la aplicación de K como fertilizante (Da Silva, Neto, Curi, \& do Vale, 1997). Para Dalbergia retusa y Hymenaea courbaril, la información sobre los requerimientos nutricionales es prácticamente nula. En leguminosas y otras plantas se ha demostrado que la cantidad y proporción de nutrimentos en el follaje se relaciona con su disponibilidad en el suelo (Chimphango, Potgieter, \& Cramer,
2015) por lo que el contenido foliar de nutrimentos se puede utilizar como un indicador de la disponibilidad de elementos que deben tener los suelos para su crecimiento óptimo. Este conocimiento podría ayudar a mejorar los proyectos de producción de especies forestales o aquellos de restauración ecológica.

Para conocer los requerimientos nutricionales de las plantas se ha utilizado el análisis de la concentración de nutrimentos en las hojas, método que se emplea con frecuencia debido a que es preciso y rápido de hacer. Además, las hojas son el punto focal donde se llevan a cabo una gran cantidad de funciones en la planta y son un indicador relativamente sensible a cambios nutricionales (Van den Driessche, 1974). Esta técnica de diagnóstico permite encontrar la proporción y cantidad de cada elemento que la planta requiere para crecer adecuadamente (Drechsel \& Zech, 1991). Además, definir niveles de deficiencia o de consumo de lujo cuando una especie excluye o acumula algún nutrimento que le podría ser tóxico en grandes cantidades (Smith \& Loneragan, 1997; Alvarado et al., 2015).

En el presente trabajo se tienen como objetivos definir el "rango satisfactorio" de concentración de nutrimentos a nivel foliar para cada uno de los elementos estudiados de cuatro fabáceas del bosque tropical estacionalmente seco de Guanacaste, Costa Rica. Además, estudiar el efecto de la capacidad de fijar nitrógeno y la tasa de crecimiento de las especies sobre la concentración foliar de nutrimentos esenciales. Se entenderá como "rango satisfactorio" el ámbito de valores máximos y mínimos de nutrimentos a nivel foliar que expresan la mayoría de los individuos de una especie en aparente crecimiento sin problemas nutricionales, excluyendo los valores extremos. Este rango se define como aquel en el que se ubican los datos obtenidos en el laboratorio entre el primer y el tercer cuartil después de eliminar los valores atípicos. De esta manera, se interpreta que si una muestra en particular tiene un valor cercano al inferior del rango, la posibilidad de que el árbol esté deficiente en el elemento es alta $>95 \%$ de 


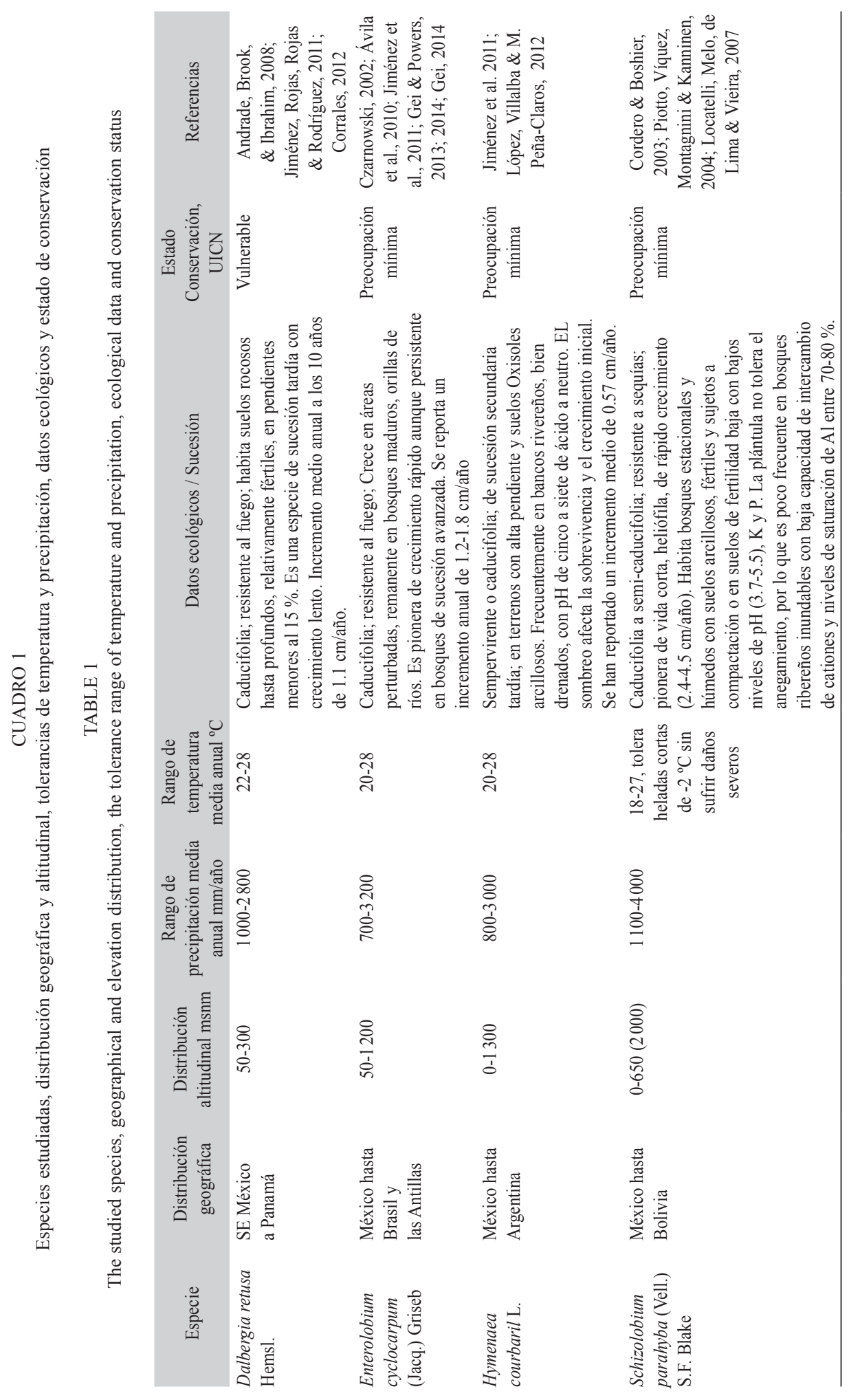


CUADRO 2

Concentración de nutrimentos en distintos componentes de la planta reportados por otros autores para las especies estudiadas

TABLE 2

Nutrients concentration in several parts of the plant reported by other authors for the four tree species studied

\begin{tabular}{|c|c|c|c|c|c|c|}
\hline \multirow{2}{*}{ Elementos } & \multirow{2}{*}{ Unidades } & \multicolumn{2}{|c|}{ E. cyclocarpum } & \multicolumn{3}{|c|}{ S. parahyba } \\
\hline & & Hojas & Madera & Biomasa aérea & Hojas & Hojarasca \\
\hline $\mathrm{N}$ & \multirow{7}{*}{$\%$} & & 0.33 & 3.1 & 2.61 & 1.65 \\
\hline $\mathrm{P}$ & & 0.21 & 0.01 & 0.31 & 0.16 & 0.12 \\
\hline K & & 1.18 & 0.44 & 1.11 & 0.18 & 0.82 \\
\hline $\mathrm{Ca}$ & & 0.66 & 0.21 & 0.68 & 2.14 & 1.99 \\
\hline $\mathrm{Mg}$ & & 0.71 & 0.06 & 0.22 & 0.21 & 0.18 \\
\hline $\mathrm{S}$ & & & 0.04 & 0.135 & 0.18 & 0.11 \\
\hline B & & 27 & 4 & 46 & & \\
\hline $\mathrm{Fe}$ & \multirow{4}{*}{$\mathrm{mg} \mathrm{kg}^{-1}$} & & 206 & 206 & & \\
\hline $\mathrm{Cu}$ & & & 6 & 8 & & \\
\hline $\mathrm{Zn}$ & & & 16 & 35 & & \\
\hline $\mathrm{Mn}$ & & & 4 & 45 & & \\
\hline Referencias & & 1 & 2 & 3 & 4 & 4 \\
\hline
\end{tabular}

1: Hunter \& Stewart, 1993; 2: Tenorio, Moya, Salas \& Berrocal, 2016; 3: AMATA S/A, 2009; 4: Corrêa, 2014.

probabilidad) y si se encuentra cerca del valor máximo del rango el riesgo de toxicidad es alto (>95\% de probabilidad).

\section{MATERIALES Y MÉTODOS}

Sitio de estudio: La investigación se realizó con muestras foliares recolectadas en diferentes localidades de la vertiente pacífica de Costa Rica (Fig. 1). El $93 \%$ del total de muestras (total: 74 muestras) se recolectó en la provincia de Guanacaste, en las localidades de Abangares, Carrillo, Hojancha, Liberia, Nadayure y Nicoya (Fig. 1). Un restante $7 \%$ de las muestras se obtuvieron en localidades más al sur de Costa Rica (Fig. 1). Las muestras de Liberia fueron recolectadas en la Estación Experimental Forestal Horizontes (EEFH) y la Finca La Flor en las llanuras de la cuenca del río Tempisque. En general, la vegetación natural de la región corresponde al bosque tropical estacionalmente seco (Jiménez, Carrillo, \& Kappelle, 2015). Se caracteriza en Guanacaste por la ocurrencia de dos períodos lluviosos, uno de mayo a junio y otro entre agosto $\mathrm{y}$ noviembre, además de dos períodos secos con humedad relativa alta, uno ventoso, largo y severo de noviembre a mayo y otro corto en julio. Los meses más calientes y secos se presentan en marzo y abril y los más fríos al final del segundo período húmedo $\mathrm{y}$ al inicio del primer período seco (Jiménez et al., 2015). En general, la vegetación de la región se caracteriza por formaciones de vegetación arbustiva, parches de vegetación sabanoide y pequeños parches de bosque caducifolio o siempreverde cerca de los ríos (Gómez, 1986). La geomorfología, geología, clima y cobertura vegetal de la región ha sido descrita por varios autores (Jiménez et al., 2015). En general, las muestras provienen de sitios ubicados a menos de 800 msnm, con regímenes de temperatura del suelo isohipertérmicos y de humedad údico y ústico.

Recolección y análisis de las muestras: En total se contó con un total de 74 muestras consistentes de 18 de $S$. parahyba, 10 de $D$. retusa, 33 de $H$. courbaril y 13 de E. cyclocarpum. Una comparación detallada de estas 


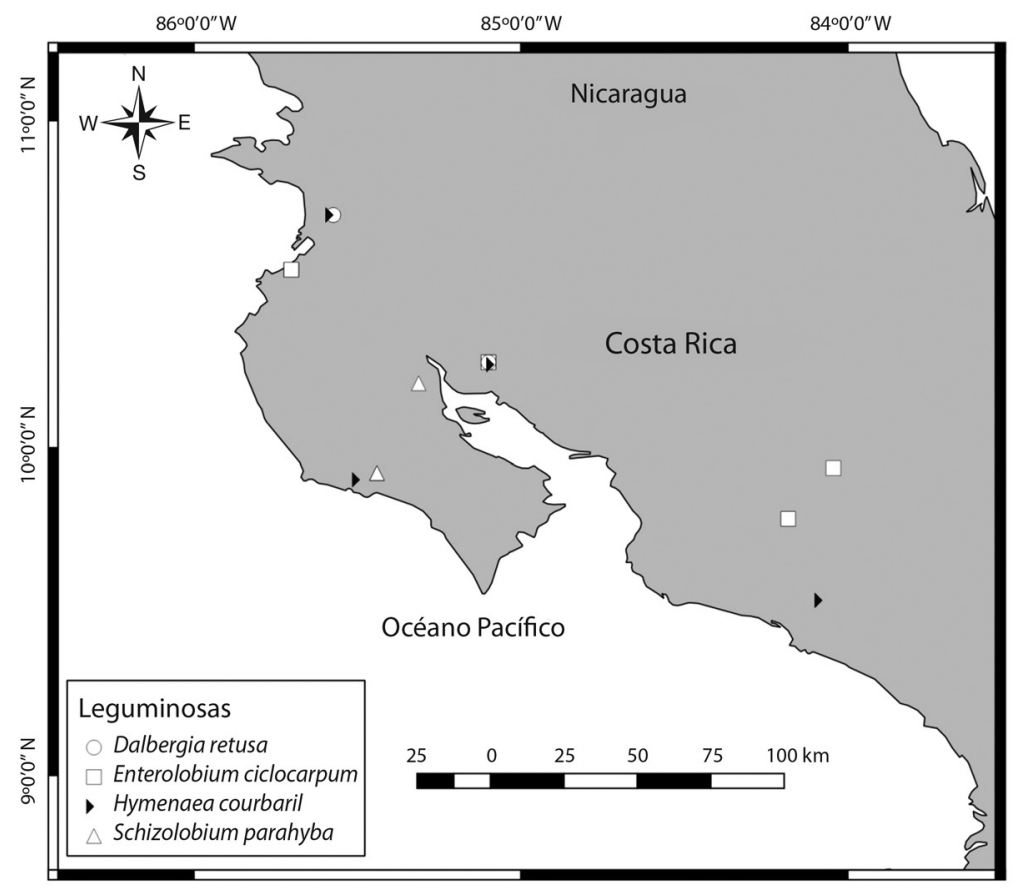

Fig. 1. Ubicación de los sitios en los que se recolectaron las muestras para cada especie de árbol estudiada.

Fig. 1. Location of sampling sites for each tree species studied.

especies se presenta en el cuadro 1. El tejido foliar utilizado se recolectó en el 2015 a partir de árboles ubicados en plantaciones y bosques de Costa Rica siguiendo los lineamientos mencionados por Bündchen et al. (2013). Con el fin de homogenizar el material vegetal se seleccionaron árboles con crecimiento vigoroso (dominantes) con alturas entre $15-20 \mathrm{~m}$ y distanciados por más de $20 \mathrm{~m}$. Se recolectó medio kilogramo de hojas frescas maduras (color verde intenso) utilizando una podadora con barra de extensión. Las hojas estuvieron totalmente expuestas a la luz, ya fuera en la parte media y terminal de la copa. Las muestras se colocaron en bolsas de papel indicando su ubicación, el nombre de la finca, la especie y el día de muestreo. La recolección de las muestras se llevó a cabo durante el período lluvioso cuando los árboles alcanzan su máximo crecimiento anual, aproximadamente un mes después de las primeras lluvias. Con el fin de evitar pérdida del material recolectado las muestras se pusieron a secar a la sombra y se transportaron al laboratorio, donde se terminaron de secar en una estufa a $70^{\circ} \mathrm{C}$ por 72 horas.

Las muestras se enviaron al Laboratorio de suelos y foliares de la Escuela de Agricultura de la Región del Trópico Húmedo (Universidad EARTH, Guácimo, Costa Rica) donde se analizaron por $\mathrm{Ca}, \mathrm{Mg}, \mathrm{K}, \mathrm{S}, \mathrm{Fe}, \mathrm{Mn}, \mathrm{Cu}, \mathrm{Zn}, \mathrm{B}$ y $\mathrm{Na}$ empleando un aparato de espectrometría de emisión siguiendo la metodología descrita por Kalra (1997) y el P por fotometría siguiendo el método del azul de molibdeno (Murphy \& Riley, 1962). La concentración de $\mathrm{N}$ se obtuvo mediante combustión seca en un analizador de $\mathrm{C}, \mathrm{H}, \mathrm{O}$ y $\mathrm{N}$ por el método de Dumas en un auto-analizador con incineración a $500{ }^{\circ} \mathrm{C}$.

Análisis estadísticos: Se estimaron los estadísticos descriptivos de la distribución de cada nutrimento para cada especie (media, error estándar, coeficiente de variación, mínimo y máximo). Para estimar diferencias entre 
los contenidos de los nutrimentos foliares entre especies se realizaron pruebas de KruskalWallis de una vía $(\alpha=0.05)$. Se utilizaron pruebas a posteriori para las comparaciones entre pares de especies. Para caracterizar y agrupar las especies de acuerdo a las relaciones de los nutrimentos foliares se usó un análisis de componentes principales (Husson, Lê \& Pagès, 2011). El análisis de componentes principales se hizo usando sólo aquellos nutrimentos en los que al menos una especie resultó diferente. Se calculó la cantidad absoluta de nutrimentos como la sumatoria de porcentajes con base a peso seco de todos los elementos en cada especie. Los rangos satisfactorios de concentración foliar para cada uno de los nutrimentos se clasificaron según los criterios previamente reportados en la literatura (Büendchen, Boeger, Reissmann, \& da Silva, 2013; Metali, Salim, Tennakoon, \& Burslem, 2015) como deficiente (por debajo del primer cuartil) y en riesgo de toxicidad o consumo de lujo (por encima del tercer cuartil) y posteriormente fueron mostrados en histogramas. Todos los análisis estadísticos se realizaron con el programa $R$ (R Core Team, 2016).
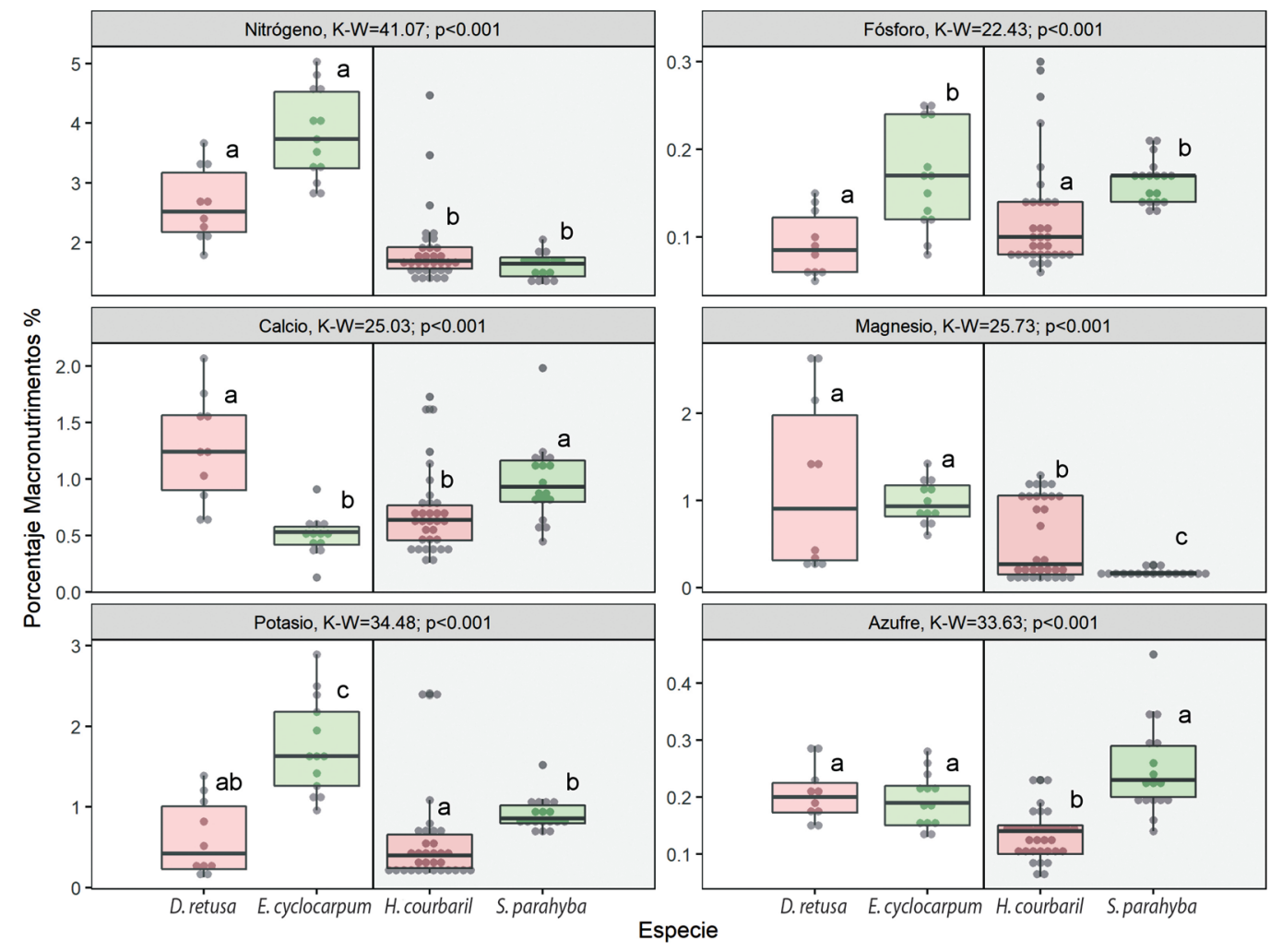

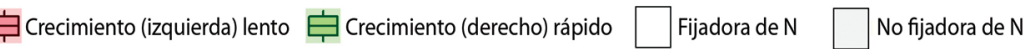

Fig. 2. Distribución de los valores de concentración de macronutrimentos en las hojas (\%) en cuatro especies de leguminosas en la vertiente pacífica de Costa Rica. Los puntos representan los valores observados, las cajas representan el intervalo intercuartil y la línea horizontal dentro de las cajas la mediana. El valor de K-W y p son respectivamente el valor del estadístico Kruskal-Wallis y la probabilidad con 3 grados de libertad. Las letras representan los grupos según las prueba de diferencias mínimas significativas.

Fig. 2. Boxplot of the foliar concentration (\%) of macronutrients in four woody legumes from de Pacific slope of Costa Rica. Within each boxplot, points represent the observed values, the box represents the interquartile range and the horizontal line represents the median. K-W and $\mathrm{p}$ are the Kruskal-Wallis statistic and the probability with 3 degrees of freedom, respectively. Letters represent the groups according to minimum differences test. 


\section{RESULTADOS}

Entre las cuatro especies comparadas se encontró variación intra e inter específica en los macronutrimentos y micronutrimentos estudiados (Fig. 2 y Fig. 3). El coeficiente de variación fue entre $13.55 \%$ en $\mathrm{N}$ de $H$. courbaril y $86 \%$ en Na de E. cyclocarpum (Cuadro 3) y en general, los coeficientes de variación más altos se notaron en los micronutrimentos (Cuadro 3). Se obtuvieron diferencias en la cantidad de nutrimentos entre las especies para la mayoría de los elementos excepto en $\mathrm{Na}$ y Fe (Fig. 3). El guanacaste, E. cyclocarpum, tuvo mayor concentración de los tres principales nutrimentos $(\mathrm{N}, \mathrm{P}$ y $\mathrm{K})$ en comparación con las otras especies (Fig. 2).

Las especies fijadoras de nitrógeno, tuvieron mayor cantidad de N, Mg (Fig. 2) y de B (Fig. 3) en comparación con las no fijadoras. Las especies de crecimiento rápido mostraron mayor cantidad de P y K (Fig. 2) y menor cantidad de Zn y Mn (Fig. 3) en comparación con las especies de crecimiento lento. $\mathrm{El} \mathrm{Ca}, \mathrm{S}, \mathrm{Na}$,

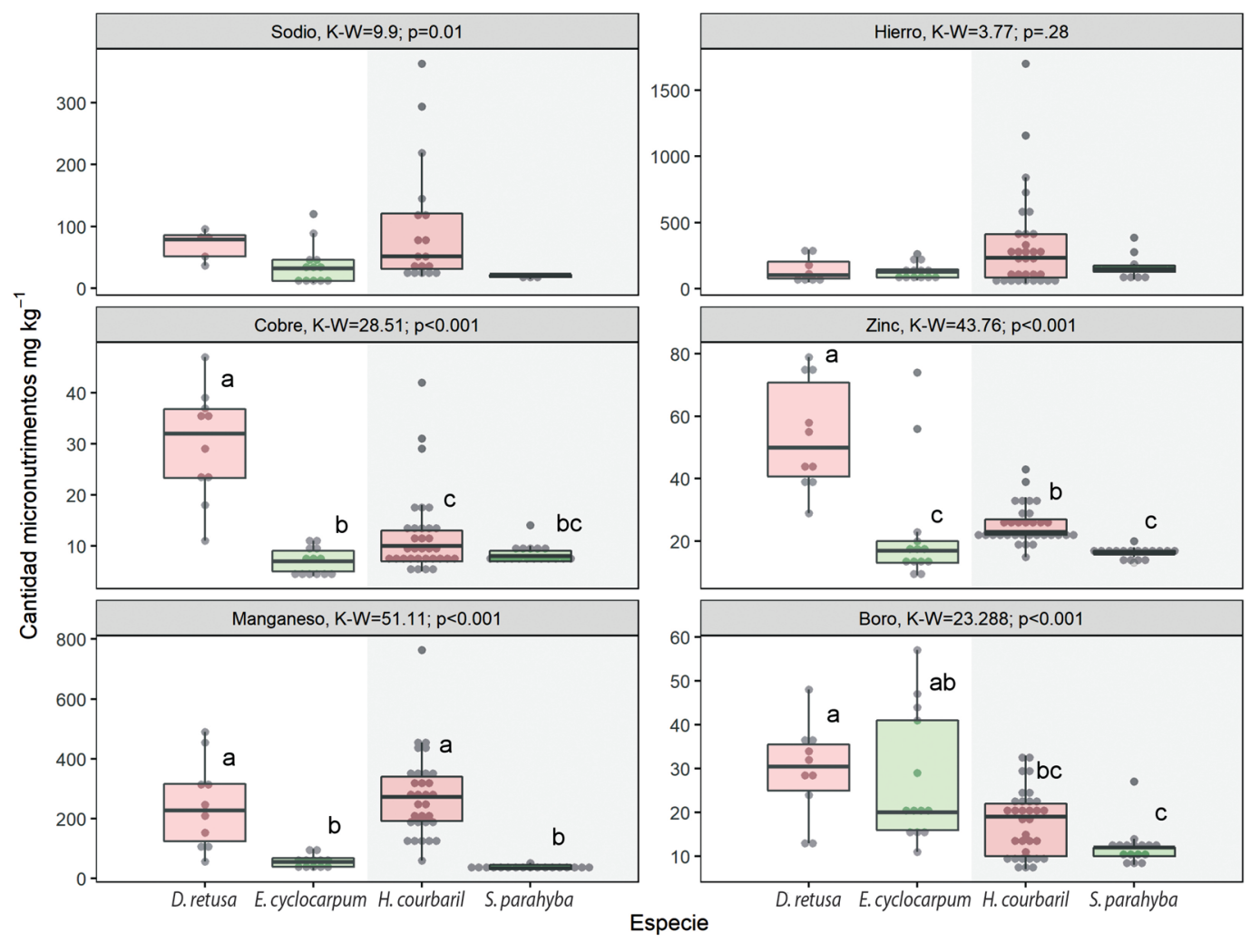

Crecimiento lento 身 Crecimiento rápido $\square$ Fijadora de $\mathrm{N} \longrightarrow$ No fijadora de $\mathrm{N}$

Fig. 3. Distribución de los valores de concentración de micronutrimentos en las hojas $\left(\mathrm{mg}^{\mathrm{k}} \mathrm{kg}^{-1}\right)$ en cuatro especies de leguminosas en la vertiente pacífica de Costa Rica. Los puntos representan los valores observados, las cajas representan el intervalo intercuartil y la línea horizontal dentro de las cajas la mediana. El valor de K-W y p son respectivamente el valor del estadístico Kruskal-Wallis y la probabilidad con 3 grados de libertad. Las letras representan los grupos según las prueba de diferencias mínimas significativas.

Fig. 3. Boxplots of the foliar concentration $(\mathrm{mg} \mathrm{kg}-1)$ of micronutrients in four woody legumes from de Pacific slope of Costa Rica. Within each boxplot, points represent the observed values, the box represents the interquartile range, and the horizontal line represents the median. K-W and p are the Kruskal-Wallis statistic and the probability with 3 degrees of freedom, respectively. Letters represent the groups according to minimum differences test. 
CUADRO 3

Estadísticos descriptivos de los nutrimentos foliares para cada especie forestal estudiada, error estándar (EE), coeficiente de variación (CV), valor mínimo (Min.) y valor máximo (Max.). Las unidades de los elementos de los macronutrimentos es porcentaje masa / masa y la de los micronutrimentos (respresentados con asterisco“*”) es mg kg ${ }^{-1}$

TABLE 3

Descriptive statistics of the foliar nutrients for each tree species studied, standard error (EE), the coefficient of variation (CV), minimum (Min.) and maximum (Max.). The units for macronutrients elements are percentage mass/mass and for micronutrients elements (marked with asterisks “*”) is $\mathrm{mg} \mathrm{kg}^{-1}$

\begin{tabular}{|c|c|c|c|c|c|c|c|c|c|c|c|c|}
\hline \multirow{2}{*}{ Parámetro } & $\mathrm{N}$ & $\mathrm{P}$ & $\mathrm{Ca}$ & $\mathrm{Mg}$ & $\mathrm{K}$ & $\mathrm{S}$ & $\mathrm{Na}^{*}$ & $\mathrm{Fe}^{*}$ & $\mathrm{Cu}^{*}$ & $\mathrm{Zn} *$ & $\mathrm{Mn}^{*}$ & $\mathrm{~B}^{*}$ \\
\hline & \multicolumn{6}{|c|}{$\%$} & \multicolumn{6}{|c|}{ * $\mathrm{mg} \mathrm{kg}^{-1}$} \\
\hline \multicolumn{13}{|c|}{ Dalbergia retusa $(\mathrm{N}=10)$} \\
\hline Media & 2.63 & 0.09 & 1.26 & 1.18 & 0.61 & 0.21 & 70 & 146.25 & 29.9 & 53.7 & 244.8 & 29.4 \\
\hline $\mathrm{EE}$ & 0.83 & 0.03 & 0.4 & 0.37 & 0.19 & 0.07 & 22.14 & 46.25 & 9.46 & 16.98 & 77.41 & 9.3 \\
\hline $\mathrm{CV}$ & 23.58 & 39.95 & 37.89 & 84.67 & 76.54 & 23.8 & 35.18 & 65.73 & 36.48 & 32.86 & 60.62 & 36.64 \\
\hline Mín. & 1.79 & 0.05 & 0.64 & 0.24 & 0.13 & 0.15 & 37 & 49 & 11 & 29 & 56 & 13 \\
\hline Máx. & 3.67 & 0.15 & 2.07 & 2.65 & 1.39 & 0.29 & 96 & 297 & 47 & 79 & 490 & 48 \\
\hline \multicolumn{13}{|c|}{ Enterolobium cyclocarpum $(\mathrm{N}=13)$} \\
\hline Media & 3.81 & 0.17 & 0.51 & 0.98 & 1.75 & 0.19 & 38.33 & 135.31 & 6.92 & 22.92 & 55.92 & 27.38 \\
\hline $\mathrm{EE}$ & 1.06 & 0.05 & 0.14 & 0.27 & 0.48 & 0.05 & 10.63 & 37.53 & 1.92 & 6.36 & 15.51 & 7.6 \\
\hline $\mathrm{CV}$ & 20.12 & 36.01 & 35.55 & 25.43 & 34.28 & 24.7 & 90.08 & 47.15 & 38.43 & 84.69 & 41.34 & 54.11 \\
\hline Mín. & 2.82 & 0.08 & 0.13 & 0.6 & 0.96 & 0.13 & 9 & 63 & 4 & 9 & 27 & 11 \\
\hline Máx. & 5.03 & 0.25 & 0.91 & 1.42 & 2.89 & 0.28 & 120 & 264 & 11 & 74 & 98 & 57 \\
\hline \multicolumn{13}{|c|}{ Hymenaea courbaril $(\mathrm{N}=33)$} \\
\hline Media & 1.87 & 0.12 & 0.71 & 0.56 & 0.59 & 0.13 & 97.33 & 325 & 12.15 & 25.24 & 277.84 & 17.67 \\
\hline $\mathrm{EE}$ & 0.32 & 0.02 & 0.12 & 0.1 & 0.1 & 0.02 & 16.94 & 56.58 & 2.12 & 4.39 & 48.37 & 3.08 \\
\hline $\mathrm{CV}$ & 33 & 52.42 & 53.12 & 83.45 & 106.19 & 32.56 & 102.68 & 111.21 & 65.97 & 23.79 & 50.11 & 42.07 \\
\hline Mín. & 1.35 & 0.06 & 0.26 & 0.08 & 0.18 & 0.06 & 20 & 40 & 5 & 15 & 59 & 7 \\
\hline Máx. & 4.47 & 0.3 & 1.73 & 1.29 & 2.41 & 0.23 & 363 & 1699 & 42 & 43 & 763 & 33 \\
\hline \multicolumn{13}{|c|}{ Schizolobium parahyba $(\mathrm{N}=18)$} \\
\hline Media & 1.62 & 0.16 & 0.98 & 0.17 & 0.92 & 0.25 & 19.8 & 161 & 8.33 & 16.24 & 36.56 & 12.06 \\
\hline $\mathrm{EE}$ & 0.38 & 0.04 & 0.23 & 0.04 & 0.22 & 0.06 & 4.67 & 37.95 & 1.96 & 3.83 & 8.62 & 2.84 \\
\hline $\mathrm{CV}$ & 13.28 & 15.57 & 35.93 & 23.27 & 21.96 & 31.88 & 19.23 & 45.18 & 20.16 & 9.37 & 22.6 & 35.62 \\
\hline Mín. & 1.3 & 0.13 & 0.45 & 0.13 & 0.66 & 0.14 & 15.4 & 72 & 7 & 13 & 26 & 8 \\
\hline Máx. & 2.05 & 0.21 & 1.98 & 0.26 & 1.52 & 0.45 & 22 & 385 & 14 & 20 & 52 & 27 \\
\hline
\end{tabular}

Fe y Cu no mostraron ningún patrón específico entre especies fijadoras o no fijadoras, o entre especies de crecimiento lento o crecimiento rápido. La concentración foliar de $\mathrm{Na}$ y $\mathrm{Fe}$ no permite utilizar estos parámetros como discriminantes ya que no varían significativamente entre las especies comparadas (Fig. 2 y Fig. 3). Por el contrario, las concentración más elevadas de $\mathrm{K}$ permiten identificar a la especie E. cyclocarpum como significativamente diferente de las demás, la concentración baja de azufre separa $H$. courbaril de las otras especies al igual que la concentración baja de Mg separa a S. parahyba de las otras especies (Fig. 2). Finalmente, la concentración alta de $\mathrm{Zn}$ y $\mathrm{Cu}$ diferencian a $D$. retusa con respecto a las otras especies (Fig. 3).

Las cinco primeras dimensiones del análisis de componentes principales explican el $84 \%$ de la variación de los valores de concentración foliar de todas las especies (Cuadro 4). El primer componente, que explica el 31 $\%$ de la variación, separa a las especies de crecimiento lento de las de crecimiento rápido y está correlacionado principalmente con los contenidos de Na, Fe y el K (Cuadro 4, Fig. 4). 
CUADRO 4

Variancia explicada por el análisis de componentes principales de los valores de macronutrimentos y micronutrimentos de cuatro especies de leguminosas forestales

TABLE 4

Variance explained by the principal component analysis for the macro- and micronutrients of four tree legume species

\begin{tabular}{lccccc} 
& Comp. 1 & Comp. 2 & Comp. 3 & Comp. 4 & Comp. 5 \\
Variancia & 3.744 & 2.483 & 1.645 & 1.397 & 0.781 \\
Porcentaje de variancia & 31.203 & 20.694 & 13.704 & 11.646 & 6.511 \\
Porcentaje de variancia acumulado & 31.203 & 51.897 & 65.601 & 77.247 & 83.758 \\
\hline
\end{tabular}
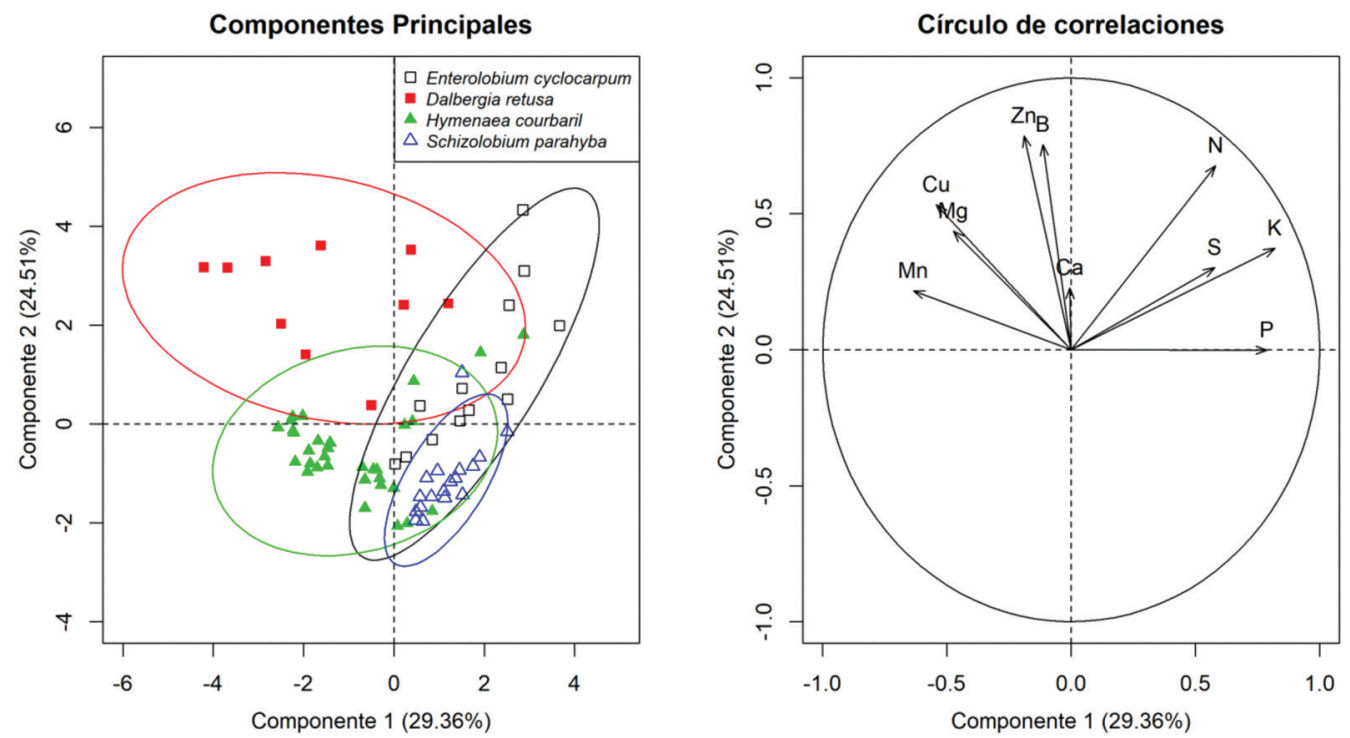

Fig. 4. Componentes principales ( 1 y 2 ) y círculo de correlaciones de los macronutrimentos y micronutrimentos de cuatro especies de leguminosas forestales. Cada color representa una especie diferente, los símbolos cuadrados son especies fijadoras de nitrógeno, los símbolos triangulares son de especies no fijadoras de nitrógeno. Lo símbolos rellenos son especies de crecimiento lento, los símbolos vacíos son especies de crecimiento rápido. Los elipses encierran el $95 \%$ de los valores. Fig. 4. Principal components ( 1 and 2 ) and correlation circle of the macro- and micronutrients of four woody legumes. Colors represent the species; squares are nitrogen-fixing species, triangles the non-nitrogen-fixing species. Filled symbols are slow-growing species and empty symbols fast-growing species. Ellipses enclose the $95 \%$ of the values.

El segundo componente, que explica el 20.6 $\%$ de la variancia, separa las especies fijadoras de $\mathrm{N}$ y las no fijadoras y está correlacionado principalmente con las concentraciones de B, Zn y N (Cuadro 4, Fig. 4). Los valores de los primeros dos componentes de E. cyclocarpum tienen trasplape con los de las otras especies, que tienen valores más diferentes entre ellas (Fig. 4). No obstante debido a que ambos componentes apenas incluyen sólo el $50 \%$ de la variación, es posible que todas las especies queden separadas al incluir otros componentes (Fig. 4).

En términos de cantidad total de concentración de macronutrimentos (sumatoria de porcentajes de todos los elementos en cada especie), esta aumenta en el orden $H$. courbaril (3.98 \%), S. parahyba (4.10\%), D. retusa $(5.98$ \%) y E. cyclocarpum (7.41\%) debido principalmente a la contribución del N. En el caso del E. cyclocarpum, este alcanza valores altos por las contribuciones del $\mathrm{N}$ y $\mathrm{K}$ y en el caso 
CUADRO 5

Secuencia de los macro and micronutimentos según la concentración foliar en cuatro especies de leguminosas estudiadas en Costa Rica

TABLE 5

Sequence of the macro- and micronutrients according to the foliar concentration in four woody legumes species studied in Costa Rica

\begin{tabular}{|c|c|}
\hline Especie & Macronutrimentos $(\%)$ \\
\hline Dalbergia retusa & $\mathrm{N}(2.63)>\mathrm{Ca}(1.26)>\mathrm{Mg}(1.18)>\mathrm{K}(0.61)>\mathrm{S}(0.21)>\mathrm{P}(0.09)$ \\
\hline Enterolobium cyclocarpum & $\mathrm{N}(3.81)>\mathrm{K}(1.75)>\mathrm{Mg}(0.98)>\mathrm{Ca}(0.51)>\mathrm{S}(0.19)>\mathrm{P}(0.17)$ \\
\hline Hymenaea courbaril & $\mathrm{N}(1.87)>\mathrm{Ca}(0.71)>\mathrm{K}(0.59)>\mathrm{Mg}(0.56)>\mathrm{S}(0.13)>\mathrm{P}(0.12)$ \\
\hline \multirow[t]{2}{*}{ Schizolobium parahyba } & $\mathrm{N}(1.62)>\mathrm{Ca}(0.98)>\mathrm{K}(0.92)>\mathrm{S}(0.25)>\mathrm{Mg}(0.17)>\mathrm{P}(0.16)$ \\
\hline & Micronutrimentos $\left(\mathrm{mg} \mathrm{kg-}^{-1}\right)$ \\
\hline Dalbergia retusa & $\mathrm{Mn}(244.8)>\mathrm{Fe}(146.25)>\mathrm{Na}(70)>\mathrm{Zn}(53.7)>\mathrm{Cu}(29.9)>\mathrm{B}(29.4)$ \\
\hline Enterolobium cyclocarpum & $\mathrm{Fe}(135.31)>\mathrm{Mn}(55.92)>\mathrm{Na}(38.33)>\mathrm{B}(27.38)>\mathrm{Zn}(22.92)>\mathrm{Cu}(6.92)$ \\
\hline Hymenaea courbaril & $\mathrm{Fe}(325)>\mathrm{Mn}(277.84)>\mathrm{Na}(97.33)>\mathrm{Zn}(25.24)>\mathrm{B}(17.67)>\mathrm{Cu}(12.15)$ \\
\hline Schizolobium parahyba & $\mathrm{Fe}(161)>\mathrm{Mn}(36.56)>\mathrm{Na}(19.8)>\mathrm{Zn}(16.24)>\mathrm{B}(12.06)>\mathrm{Cu}(8.33)$ \\
\hline
\end{tabular}

de D. retusa por las contribuciones del $\mathrm{Mg}$ y Ca. La cantidad total de macronutrimentos fue más alta en especies fijadoras que en especies no fijadoras de $\mathrm{N}$ y más alta en especies de crecimiento rápido que en especies de crecimiento lento (Cuadro 5).

Los valores más elevados de concentración foliar de nutrimentos en todas las especies fueron los de $\mathrm{N}$ y los más bajos los del $\mathrm{P}$ (Cuadro 5). Según la secuencia de la concentración foliar de los diferentes elementos en orden descendente para las especies estudiadas se infiere que la concentración foliar de los macronutrimentos para la mayoría de las especies en estudio sigue el orden $\mathrm{N}>\mathrm{Ca}>\mathrm{K}>$ $\mathrm{Mg}>\mathrm{S} \geq \mathrm{P}$. Excepto para E. cyclocarpum cuya concentración de K es mayor a la del Ca y para $S$. parahayba en el que la concentración de $\mathrm{S}$ es mayor que la de $\mathrm{Mg}$. La concentración foliar de los micronutrimentos siguió el mismo orden en las especies no fijadoras de $\mathrm{N}$ (H. courbaril y S. parahyba). Pero el orden cambió en las otras especies aunque el $\mathrm{Mn}$ y $\mathrm{Fe}$ siempre fueron más altos que el $\mathrm{Na}$ y el $\mathrm{Zn}$, y el Cu y B siempre fueron más bajos que el $\mathrm{Na}$.

Rangos Satisfactorios (RS) de Concentración Foliar: Empleando como criterio de definición del rango satisfactorio los valores de concentración para los percentiles 25 (mínimo) y 75 (máximo), se encontró que $D$. retusa y $H$. courbaril podrían requerir menor cantidad de nutrimentos como fósforo o potasio que $E$. cyclocarpum y $S$. parahyba, que tienen más requerimientos nutricionales (Cuadro 6). Relativo a su capacidad de fijar nitrógeno $D$. retusa y $H$. courbaril también tuvieron menores concentraciones de este macronutrimento que $E$. cyclocarpum y $S$. parahyba (Cuadro 6).

\section{DISCUSIÓN}

A pesar de que se considera que cierta proporción de los nutrimentos presentes en las hojas se deben a un componente genético (Masunaga, Kubota, Hotta \& Wakatsuki, 1998, Masunaga et al., 1998; Fyllas et al., 2009), otros factores (p.e. edáficos) pueden afectar la concentración foliar de los nutrimentos en el follaje. Chimphango et al. (2015) encontraron que en los hábitats con especies leguminosas en suelos derivados de calizas y areniscas tienen más $\mathrm{N}$ total, $\mathrm{P}, \mathrm{K}, \mathrm{Mg}, \mathrm{Na}$ y $\mathrm{C}$ pero solamente de $\mathrm{C}$ en los derivados de granito en Sudáfrica. Lo anteriormente mencionado indica empíricamente una relación entre la concentración foliar y las propiedades químicas de los suelos y que algunas especies puedan requerir suelos fértiles mientras que otras enriquecen el medio particularmente con $\mathrm{N}$ y Mg (Van Breugel et al., 2011). 
CUADRO 6

Rango satisfactorio (RS) de concentración foliar de elementos (valores en los percentiles 25 y 75) en D. retusa, E. cyclocarpum, H. courbaril y $S$. parahyba en Guanacaste, Costa Rica

TABLE 6

Satisfactory range (RS) of foliar concentration of elements (interquartile range) for D. retusa, E. cyclocarpum, H. courbaril and S. parahyba in Guanacaste, Costa Rica

\begin{tabular}{|c|c|c|c|c|}
\hline Elemento & Dalbergia retusa & Enterolobium cyclocarpum & Hymenaea courbaril & Schizolobium parahyba \\
\hline \multicolumn{5}{|c|}{ Macronutrimentos $(\%)$} \\
\hline $\mathrm{N}$ & $2.18-3.17$ & $3.24-4.52$ & $1.56-1.92$ & $1.43-1.75$ \\
\hline $\mathrm{Ca}$ & $0.90-1.56$ & $0.42-0.58$ & $0.46-0.77$ & $0.80-1.16$ \\
\hline $\mathrm{K}$ & $0.23-1.01$ & $1.26-2.18$ & $0.24-0.66$ & $0.8-1.02$ \\
\hline $\mathrm{Mg}$ & $0.31-1.97$ & $0.82-1.17$ & $0.15-1.05$ & $0.15-0.18$ \\
\hline $\mathrm{S}$ & $0.17-0.22$ & $0.15-0.22$ & $0.10-0.15$ & $0.20-0.29$ \\
\hline $\mathrm{P}$ & $0.06-0.12$ & $0.12-0.24$ & $0.08-0.14$ & $0.14-0.17$ \\
\hline \multicolumn{5}{|c|}{ Micronutrimentos $\left(\mathrm{mg} \mathrm{kg}^{-1}\right)$} \\
\hline $\mathrm{Na}$ & $52-86$ & $12-46$ & $31-121$ & $19-22$ \\
\hline $\mathrm{Fe}$ & $79-205$ & $86-148$ & $84-413$ & $128-176$ \\
\hline Mn & $124-316$ & $39-67$ & $191-340$ & $31-45$ \\
\hline $\mathrm{Zn}$ & $41-71$ & $13-20$ & $22-27$ & $16-17$ \\
\hline $\mathrm{B}$ & $25-36$ & $16-41$ & $10-22$ & $10-12$ \\
\hline $\mathrm{Cu}$ & $23-37$ & $5-9$ & $7-13$ & $7-9$ \\
\hline
\end{tabular}

Los altos valores en los coeficientes de variación encontrados en el presente estudio se pueden deber a un efecto de sitio y a las diferentes edades de los árboles muestreados (Letcher et al., 2015). Esto se puede observar en nutrimentos como el Fe, Mn y $\mathrm{Na}$ en los que no hay una diferencia entre las especies. La distribución de frecuencias para cada especie y para muchos de los elementos analizados no tiende a la normalidad (datos no incluidos), probablemente debido al tamaño de la muestra. Además, los valores de concentración foliar bajos son más abundantes que los altos (Alvarado et al., 2015), presentándose distribuciones sesgadas hacia la izquierda como ha sido reportado en bosques de Europa (Vanmechelen, Groenemans \& van Ranst, 1997) y subtropicales lluviosos de Taiwan (Wu, Tsui, Hseih, Asio, \& Chen, 2007).

Se observó mayor concentración de nutrimentos en las especies fijadoras que en las no fijadoras de $\mathrm{N}$ y además dentro de cada uno de esos grupos, que las de crecimiento rápido tenían mayor concentración de nutrimentos que las de crecimiento lento. El efecto de "dilución" de la concentración foliar puede ayudar a explicar las disminuciones en concentración foliar en especies de crecimiento rápido en las que la mayor concentración de un elemento (ej. N) resulta en un incremento de biomasa foliar mientras que al mismo tiempo la concentración los otros nutrimentos por unidad de biomasa se disminuye/reduce (Marschner, 1995). Igualmente otros factores que afectan la concentración foliar de algunos elementos son el lavado (ej. K), otras interacciones de naturaleza fisiológica que envuelven cambios en su translocación y las interacciones que ocurren en la superficie de las raíces o en su membrana celular (Van den Driessche, 1974). En el caso de $S$. parahyba, aunque es la única especie considerada pionera de las comparadas en el presente estudio, su contenido total de nutrimentos en el tejido foliar no es el más elevado, como sugiere la hipótesis de sucesión ecológica descrita por Broadbent et al. (2014).

Los valores más elevados de concentración foliar de nutrimentos en todas las especies fueron los de N (Cuadro 3 y Cuadro 5). En las especies D. retusa y E. cyclocarpum esto 
podría estar asociado a la fijación simbiótica del elemento con bacterias del género Rhizobium (Gei, 2014; Gei \& Powers, 2013; 2014) mientras que las concentraciones foliares de $\mathrm{P}$ y $\mathrm{Cu}$ en las especies estudiadas son muy bajas como ha sido mencionado para un gran número de especies latifoliadas tropicales (Drechsel \& Zech, 1991). Sprent (1999) discute la necesidad de $\mathrm{P}$ en leguminosas fijadoras y no fijadoras de $\mathrm{N}$ y concluye que no se comprueba que en los trópicos se tengan los altos requerimientos del elemento mencionados en regiones subtropicales, encontrándose leguminosas que pueden fijar el elemento en suelos bajos en P disponible. Büendchen et al. (2013) indican que las altas concentraciones foliares de $\mathrm{N}$ y las bajas de $\mathrm{P}$ redundan en una eficiencia inversa de uso de estos nutrimentos, es decir la del $\mathrm{N}$ es baja y la del P elevada. Para $H$. coubaril en Honduras se menciona que este hecho podría asociarse a la presencia de micorrizas ligadas a su sistema radical (Hunter \& Stewart, 1993) lo que concuerda con la poca respuesta de esta especie a la adición de $\mathrm{P}$ en invernadero (Resende et al., 1999; Santos, Resende, Neto \& Corte, 2008).

La concentración foliar de $\mathrm{Ca}$ observada en todas las especies está dentro de los valores del rango normal para especies tropicales latifoliadas, con una relación $\mathrm{Ca} / \mathrm{K}$ mayor que la unidad (Alvarado, 2015). Lo anterior podría interpretarse como adaptación genética a ambientes tropicales estacionalmente secos en los que la disponibilidad de $\mathrm{Ca}$ en el suelo es elevada. Los valores de concentración foliar de los micronutrimentos se encuentran dentro de los considerados normales para Fe y Mn para otras especies forestales, al igual que sucede con las concentraciones foliares de $\mathrm{Na}, \mathrm{Zn}$ y B (Stone, 1990; Reeves \& Baker, 2000).

En el cuadro 5 se muestra la secuencia de la concentración foliar de los diferentes elementos en orden descendente para las especies estudiadas. Se infiere que la concentración foliar de los macronutrimentos para todas las especies sigue el orden $\mathrm{N}>\mathrm{Ca}>\mathrm{Mg}>\mathrm{K}>$ $\mathrm{S} \geq \mathrm{P}$ es una tendencia que domina en especies forestales tropicales latifoliadas (Drechsel \& Zech, 1991; Alvarado et al., 2015b). La concentración foliar de los micronutrimentos en orden descendente sigue el orden $\mathrm{Fe}>\mathrm{Mn}$ $>\mathrm{Na}>\mathrm{B}>\mathrm{Zn}>\mathrm{Cu}$. Aunque los valores de $\mathrm{Na}, \mathrm{B}$ y Zn pueden variar de posición según la especie sin que se cuente con otras experiencias similares en la literatura que permitan explicar el porqué de esta relación. En el presente trabajo se suministra información sobre $\mathrm{Na}$ y B elementos raras veces reportados para estas especies en la literatura.

\section{Rangos satisfactorios de concentración}

foliar: El empleo del análisis foliar en el sector forestal es limitado entre otras razones por la falta de criterios de interpretación de los resultados emanados de los laboratorios de servicio (Alvarado, 2015). Empleando como criterio de definición de los rangos satisfactorios como los valores de concentración de nutrimentos entre los percentiles 25 (mínimo) y 75 (máximo), se recomienda utilizar los valores presentados en el cuadro 6 para interpretar los resultados de análisis foliares de estas especies del bosque tropical estacionalmente seco de Costa Rica. Los resultados obtenidos en el análisis de este trabajo son un aporte al conocimiento sobre las concentraciones foliares de las especies $D$. retusa e $H$. courbaril para las que no existen estudios previos que describan la concentración foliar de nutrimentos en la vertiente pacífica estacionalmente seca de Costa Rica.

\section{AGRADECIMIENTOS}

Los autores expresan su agradecimiento al personal de apoyo de campo en la Finca La Flor y en el laboratorio de suelos de la EARTH por la colaboración prestada en el proceso de recolección de muestras y su posterior análisis químico en el laboratorio. También se agradece la colaboración prestada por el personal de la E.E. Forestal Horizontes durante el muestreo foliar en sus plantaciones.

\section{RESUMEN}

La productividad de las plantaciones forestales con especies nativas se puede incrementar si se conocen bien 
los requerimientos nutricionales de cada una de las especies. Estos requerimientos pueden variar de acuerdo a las estrategias ecológicas de las especies y a las adaptaciones que tienen, como por ejemplo la capacidad de fijar nitrógeno. En el presente trabajo se presentan los "rangos satisfactorios" de concentración foliar de macronutrimentos y micronutrimentos para cuatro leguminosas del trópico estacionalmente seco de Guanacaste, Costa Rica. Se analizó la concentración foliar de N, P, Ca, Mg, K, S, Fe, Mn, $\mathrm{Cu}, \mathrm{Zn}$ y B, Na en las especies Dalbergia retusa (cocobolo), Enterolobium cyclocarpum (guanacaste), Hymenaea courbaril (guapinol) y Schizolobium parahyba (gallinazo). La concentración de nutrimentos varió con las especies y según la capacidad de fijar nitrógeno o no y dentro de cada una de esas categorías de acuerdo a la tasa de crecimiento. La concentración total de nutrimentos fue mayor para las especies fijadoras de $\mathrm{N}$ que para las no fijadoras y dentro de cada uno de esos grupos la concentración total de nutrimentos fue mayor para las especies de crecimiento rápido comparado con aquellas de crecimiento más lento. De acuerdo a la cantidad de nutrimentos observados en las hojas, se recomienda que $H$. courbaril y $D$. retusa deberían ser sembradas en suelos relativamente pobres de nutrimentos, mientras que E. cyclocarpum y $S$. parahyba en suelos más fértiles. Para $D$. retusa e $H$. courbaril se presenta información nueva sobre concentraciones y se mejora la misma para algunos elementos no mencionados en la literatura para E. cyclocarpum y S. parahyba.

Palabras clave: análisis foliar; trópico estacionalmente seco; Dalbergia retusa; Enterolobium cyclocarpum; Hymenaea courbaril y Schizolobium parahyba.

\section{REFERENCIAS}

Alvarado, A. (2015). Plant nutrition in tropical forestry. In L. Pancel \& M. Köhl (Eds.), Tropical Forestry Handbook (Volume 2, pp. 1113-1202). Springer-Verlag: Berlin Heidelberg.

Alvarado, A., Camacho, G., Fernández-Moya, M. E., Mezger, J., Mata, R., Bertsch, F., ... Vaides, E. E. (2015). Interpretación del análisis foliar de varias especies forestales latifoliadas del trópico americano. VIII Congreso Nacional de Suelos, San José, Costa Rica.

Andrade, H. J., Brook, R., \& Ibrahim, M. (2008). Growth, production and carbon sequestration in silvopastoril systems with native timber species in the dry lowlands of Costa Rica. Plant Soil, 308, 11-22.

AMATA S/A. (2009). Revisão sobre paricá: Schizolobium amazonicum Huber ex Ducke.

Ávila, Y., Ballón-Falcón, C., Bustamante, K., CondoriCaballón, C., Rosero-Alvarado, J., \& Custodio, C. (2010). Growth evaluation in diameter in Enterolobium cyclocarpum's trees (Jacq) Griseb (Oreja de negro) by dendrochronlogy analysis. IX Congreso Nacional Forestal. Huancayo, Perú.

Broadbent, E. N., Almeida, A. M., Asner, G. P., Soriano, M., Field, C. B., Ramos, H., ... Giles, L. (2014). Intergrating stand and soil properties to understand foliar nutrient dynamics during forests succession following slash-and-burn agriculture in the Bolivian Amazon. PLoS-ONE, 9(2), e86042. doi:10.1371/ journal.pone.0086042

Büendchen, M., Boeger, M. R. T., Reissmann, C. B., \& da Silva, S. L. C. (2013). Nutritional status and nutrient use efficiency in tree species of subtropical forest in southern Brazil. Scientia Forestalis, 41, 227-236.

Chimphango, S. B. M., Potgieter, G., \& Cramer, M. D. (2015). Differentiation of the biogeochemical niches of legumes and non-legumes in the Cape Floristic Region of South Africa. Plant Ecology, 216, 1583-1595.

Cordero, J. \& Boshier, D. H. (2003). Árboles de Centroamérica. Un manual para extensionistas. Turrialba: Centro Agronómico Tropical de Investigación y Enseñanza (CATIE).

Corrales, J. (2012). Determinación de ecuaciones de volumen y coeficiente mórfico para la especie Dalbergia retusa, en la zona de Parrita, Puntarenas, Costa Rica (Tesis de Licenciatura). Instituto Tecnológico de Costa Rica, Costa Rica.

Corrêa, F. L. (2014). Ciclagem de nutrientes em sistema agroflorestal com especies frutiferas e florestais de Rondonia, Brasiln (Tesis de Doctorado). Universidad Federal de Lavras, Minas Gerais.

Czarnowski, M. (2002). Suitability of native species for reforestation in the tropical dry forest of Costa Rica, Estación Experimental Forestal Horizontes, Área de Conservación Guanacaste, CR. Georg-August University.

Da Silva, I. R., Neto, A. E. F., Curi, N. \& do Vale, F. R. (1997). Crescimento inicial de quatorze espécies florestais nativas em resposta à adubação potássica. Pesquisa Agropecuária Brasileira, 32, 205-212.

Drechsel, P. \& Zech, W. (1991). Foliar nutrient levels of broad-leaved tropical trees: A tabular review. Plant Soil, 131, 29-46.

Fyllas, N. M., Patiño, S., Baker, T. R., Bielefeld, G., Martinelli, L. A., Quesada, C. A., ... Lloyd, J. (2009). Basin-wide variations in foliar properties of Amazonian forest: phylogeny, soils and climate. Biogeosciences, 6, 2677-2708.

Gei, M. G. (2014). Biological nitrogen fixation in tropical dry forests of Costa Rica: patterns and controls (Tesis de doctorado). University of Minnesota. 
Gei, M. G. \& Powers, J. S. (2013). Do legumes and nonlegumes tree species affect soil properties in unmanaged forests and plantations in Costa Rican dry forests? Soil Biology \& Biochemistry, 57, 264-272.

Gei, M. G. \& Powers, J. (2014). Nutrient cycling in tropical dry forests. In A. Sanchez-Azofeifa, J. S. Powers, G. W. Fernandes, \& M. Quesada (Eds.), Tropical Dry Forests in the Americas: Ecology, Conservation, and Management (pp. 141-156). CRC Press: Boca Ratón, Florida.

Gillespie, T. W., Grijalva, A., \& Farris, C. N. (2000). Diversity, composition, and structure of tropical dry forest in Central America. Plant Ecology, 147, 37-47.

Gómez, L. (1986). Vegetación de Costa Rica. San José, Costa Rica: Editorial de la Universidad Estatal a Distancia.

Hunter, I. \& Stewart, J. (1993). Foliar nutrient and nutritive content of Central American multipurpose tree species growing at Comayagua, Honduras. Commonwealth Forestry Review, 72, 193-197.

Husson, F., Lê, S. \& Pagès, J. (2011). Exploratory multivariate analysis by example using $R$. Boca Ratón, Florida: CRC Press.

Jacobs, B. F. (2004). Palaeobotanical studies from tropical Africa : relevance to the evolution of forest, woodland and savannah biomes. Philosophical Transactions: Biological Science, 359, 1573-1583.

Jiménez, Q., Carrillo, E. \& Kappelle, M. (2015). The northern pacific lowland seasonal dry forest of Guanacaste and the Nicoya Peninsula. In M. Kappelle (Ed.), Costa Rican Ecosystems (pp. 247-289). Chicago: University of Chicago Press.

Jiménez, Q., Rojas, F. E., Rojas, V., \& Rodríguez, L. (2011). Árboles Maderables de Costa Rica, Ecología y Silvicultura. Santo Domingo, Heredia: Editorial INBio.

Kalra, Y. (1997). Handbook of reference methods for plant analysis. Boca Ratón, Florida: CRC Press.

Letcher, S. G., Lasky, J. R., Chazdon, R. L., Norden, N., Wright, S. J., Meave, J. A., ... Williamson, G. B. (2015). Environmental gradients and the evolution of successional habitat specialization: a test case with 14 Neotropical forest sites. Journal of Ecology, 103, 1276-1290.

Lewis, G. P. (2005). Legumes of the world. Royal Botanic Gardens. Kew, Inglaterra.

Locatelli, M., Melo, A. S., de Lima, L. M. L., \& Vieira, A. H. (2007). Deficiências nutricionais em mudas de Schizolobium parahyba var amazonicum. Revista Brasileira de Biociências, 5, 648-650.
López, L., Villalba, R., \& Peña-Claros, M. (2012). Ritmos de crecimiento diamétrico en los bosques secos tropicales: aportes al manejo sostenible de los bosques de la provincia biogeográfica del Cerrado Boliviano. Bosque (Valdivia), 33, 21-22.

Masunaga, T., Kubota, D., Hotta M. \& Wakatsuki, T. (1998). Nutritional characteristics of mineral elements in leaves of tree species in tropical rain forest, West Sumatra, Indonesia. Soil Science and Plant Nutrition, 44, 315-329.

Masunaga, T., Kubota, D., William, U., Hotta, M., Shinmura, Y., \& Wakatsuki, T. (1998). Spatial distribution pattern of trees in relatlon to soil edaphic status in tropical rain forest in West Sumatra, Indonesia I. Distribution of accumulating trees. Tropics, 7, 209-222.

Marschner, H. (1995). Mineral nutrition of higher plants (Segunda edición). Londres, Inglaterra: Academic Press.

Menge, D. N. L. \& Chazdon, R. L. (2016). Higher survival drives the success of nitrogen-fixing trees through succession in Costa Rican rainforests. New Phytologist, 209, 965-977.

Metali, F, Abu Salim, K., Tennakoon, K., \& Burslem, D. F. (2015). Controls on foliar nutrient and aluminium concentrations in a tropical tree flora: phylogeny, soil chemistry and interactions among elements. New Phytologist, 205, 280-292.

Murphy, J. \& Riley, J. P. (1962). A modified single solution method for the determination of phosphate in natural waters. Analytica Chimica Acta, 27, 31-36.

Piotto, D., Víquez, E., Montagnini, F., \& Kanninen, M. (2004). Pure and mixed forest plantations with native species of the dry tropics of Costa Rica: A comparison of growth and productivity. Forest Ecology and Management, 190, 359-372.

R Development Core Team. (2016). R: A Language and Environment for Statistical Computing. Viena, Austria.

Reeves, R. \& Baker, A. (2000). Metal-accumulating plants. In I. Raskin \& B. D. Ensley (Eds.), Phytoremedation of Toxic Metals: Using Plants to Clean up Environments (pp. 193-229). John Wiley \& Sons Inc.: New York, USA.

Resende, A. V., Neto, A. E., \& Curi, N. (2005). Mineral nutrition and fertilization of native tree species in Brazil. Journal of Sustainable Forestry, 20, 45-81.

Resende, A. V., Neto, A. E., Muniz, J. A., Curi, N., \& Faquin, V. (1999). Crescimento inicial de espécies florestais de diferentes grupos sucessionais em resposta a doses de fósforo. Pesquisa Agropecuária Brasileira, 34, 2071-2081. 
Santos, J. Z. L., Resende, A. V., Neto, A. E., \& Corte, E. F. (2008). Crescimento, acúmulo de fósforo e frações fosfatadas em mudas de sete espécies arbóreas nativas. Revista Árvore, 32, 799-807.

Smith, F. \& Loneragan, J. (1997). Interpretation of plant analysis: concepts and principles. In D. J. Reuter \& J. B. Robinson (Eds.), Plant Analysis and Interpretation Manual (pp. 35-51). Collingwood, Vic.: CSIRO Publishing.

Sprent, J. I. (1999). Not all nitrogen-fixing legumes have a high requirement for phosphorus. Agroforestry Forum, 9, 17-20.

Stone, E. L. (1990). Boron deficiency and excess in forest trees: A review. Forest Ecology and Management, $37,49-75$.

Tenorio, C., Moya, R., Salas, C., \& Berrocal, A. (2016). Evaluation of wood properties from six native species of forest plantations in Costa Rica. Bosque (Valdivia), $37,71-84$.

Van Breugel, M., Hall, J. S., Craven, D. J., Gregoire, T. G., Park, A., Dent, D. H., ... Ashton, M. S. (2011). Early growth and survival of 49 tropical tree species across sites differing in soil fertility and rainfall in Panama. Forest Ecology and Management, 261, 1580-1589.

Van den Driessche, R. (1974). Prediction of mineral nutrient status of trees by foliar analysis. The Botanical Review, 40, 247-394.

Vanmechelen, L., Groenemans, R., \& van Ranst, E. (1997). Forest Soil Condition in Europe, Results of a Largescale Soil Survey. EC-UN/ECE. Bruselas, Bélgica.

Wu, C. C., Tsui, C. C., Hseih, C. F., Asio, V. B., \& Chen, Z. S. (2007). Mineral nutrient status of tree species in relation to environmental factors in the subtropical rain forest of Taiwan. Forest Ecology and Management, 239, 81-91. 